$\mathrm{KD}$ is currently unknown, according to the available literature and our experiences, it is important to point out that therapy for $\mathrm{KD}$ also leads to the resolution of lung problems and findings.

\section{ASSOCIATION BETWEEN GASTROINTESTINAL MANIFESTATIONS AND THE RISK OF RENAL DISEASE IN CHILDREN WITH IGA VASCULITIS}

\begin{abstract}
${ }^{1}$ Mario Sestan*, ${ }^{1}$ Nastasia Kifer, ${ }^{2}$ Sasa Srsen, ${ }^{3}$ Aleksandar Ovuka, ${ }^{4}$ Mateja Batnozic Varga, ${ }^{4}$ Matej Sapina, ${ }^{1}$ Martina Held, ${ }^{1}$ Maja Ban, ${ }^{1}$ Ana Kozmar, ${ }^{1}$ Marijana Coric, ${ }^{1}$ Stela Bulimbasic, ${ }^{1}$ Kristina Crkvenac, ${ }^{1}$ Danko Milosevic, ${ }^{1}$ Marijan Frkovic, ${ }^{5}$ Alenka Gagro, ${ }^{1}$ Marija Jelusic. ${ }^{1}$ University Hospital Centre Zagreb, University of Zagreb, School of Medicine, Zagreb; ${ }^{2}$ University Hospital Centre Split, University of Split, School of Medicine, Split; ${ }^{3}$ University Hospital Centre Rijeka, University of Rijeka, School of Medicine, Rijeka; ${ }^{4}$ University Hospital Centre Osijek, University of Osijek, School of Medicine, Osijek; ${ }^{5}$ Children's Hospital Zagreb, Zagreb; University of Osijek, School of Medicine, Osijek, Croatia
\end{abstract}

\subsection{6/archdischild-2021-europaediatrics.451}

Indroduction $\operatorname{IgA}$ vasculitis (IgAV) is the most common childhood-vasculitis in which more than $50 \%$ of children develop gastrointestinal (GI) symptoms. In 10-20\% of patients serious complications such as intussusception, bowel perforation, and massive bleeding may occur. The most important complication is the development of nephritis with progression to chronic renal failure in about 3\% of children. The aim of the research was to analyze clinical and biochemical parameters in patients with IgAV and GI manifestations.

Methods This retrospective study included children with IgAV reviewed in five Croatian University Centers for pediatric rheumatology in the period 2009 to 2019.

Results Out of 611 children with IgAV, 320 were males and 291 were females. The overall GI symptoms prevalence was $45.9 \%$ and the median (range) age at diagnosis was 6.42 (4.58.83) years. Among patients with GI symptoms there were 1.44 times more males $(\mathrm{N}=166)$ than females $(\mathrm{N}=115)$, which was statistically significant $(p=0.003)$. Patients with GI symptoms had less infections before the appearance of purpura $(59.8 \%$ vs. $70.9 \%, p=0.005)$ and were found to be significantly more likely to have rash distributed on the trunk $(61.9 \%$ vs. $48.5 \%, \mathrm{p}=0.001)$, and upper extremities $(35.2 \%$ vs. $24.7 \%, \mathrm{p}=0.006)$, as well as generalized rash $(38.8 \%$ vs. $28.3 \%, \mathrm{p}=0.008)$. These patients also had significantly higher values of C-reactive protein, leukocyte count, erythrocytes and platelets, hemoglobin, hematocrit and D-dimer concentrations and lower levels of $\operatorname{IgG}$ and $\operatorname{IgM}$. In our cohort 42 out of 281 children (14.9\%) had the most severe GI manifestations (intussusception and/or massive GI bleeding) with significantly higher values of 24-hour urine protein levels and D-dimer concentrations and lower total serum protein, albumin, IgG, IgM and C3 levels in comparison with children whose GI manifestations were not severe. Predictors of severe GI involvement were: relapse of the disease, generalized rash, rash extended on upper extremities, rash extended to the face, recurrent rash and renal involvement, as well as lower values of prothrombin time, fibrinogen and $\operatorname{IgM}$ among the laboratory parameters. Patients with GI symptoms were 1.68 times more likely to develop nephritis, and this probability was 2.58 times higher if GI symptoms occurred before other symptoms. Other predictors of nephritis were: severe and moderate GI manifestations, recurrent rash, one or more relapses of IgAV, and older age.

Conclusion Older children with IgAV and severe GI manifestations in whom IgAV begun with GI symptoms had a higher risk of acute and chronic complications of the disease.

SUPPORT: Croatian Science Foundation project IP-2019-048822

\section{THE IMPACT OF SYSTEMIC IMMUNOMODULATORY THERAPY ON THE INTRAOCULAR INFLAMMATION AND THE NEED FOR TOPICAL GLUCOCORTICOID THERAPY IN PATIENTS WITH JUVENILE IDIOPATHIC ARTHRITIS- ASSOCIATED UVEITIS} ${ }^{1}$ Martina Galiot Delic, 'Sonja Jandrokovic, ${ }^{2}$ Martina Held, ${ }^{2}$ Marijan Frkovic, ${ }^{2}$ Marija Jelusic, ${ }^{1}$ Nenad Vukojevic. 'University Hospital Centre Zagreb, Department of Ophthalmology, University of Zagreb School of Medicine; ${ }^{2}$ University Hospital Centre Zagreb, Department of Pediatrics, University of Zagreb School of Medicine, Zagreb, Croatia

10.1136/archdischild-2021-europaediatrics.452

Indroduction Juvenile idiopathic arthritis associated uveitis (JIA-U) is the most common and potentially most destructive extraarticular manifestation of JIA. The aim of this research was to determine the need for topical glucocorticoid therapy (TGC) in patients with JIA-U on systemic biological therapy in comparison to patients treated with methotrexate (MTX) only.

Methods We have conducted a longitudinal observational study with JIA-U patients in whom systemic immunomodulatory treatment (IMT: biologics and/or MTX) was introduced and who were followed at least 3 months in the period between 2011 and 2017. The data about the number of cells in the anterior chamber (AC) according to Standardization of Uveitis Nomenclature (SUN) Working Group criteria, related to TGC, systemic therapy and JIA complications were collected during each examination. Generalized linear mixed models were used to analyze the relationships between treatment with biologics, MTX, TGC and the grade of inflammation in AC according to SUN criteria.

Results 38 JIA-U patients (69 eyes) with median (range) age of 4.9 (2-15) years and follow up period of 209 (19-381) weeks were included. There were a total of 1205 examinations. At the first examination JIA-U was detected in 16 $(42.1 \%)$ of patients, $59(79.7 \%)$ of the eyes had $\leq 1+$ cells in the AC, and in 19 (50\%) of JIA-U patients complications were already present. MTX was introduced in $23(60.5 \%)$ JIA-U patients before the initiation of the study. $8(21 \%)$ had already received biologics, while in $4(10.5 \%)$ prior systemic glucocorticoids were also used. Before the completion of the study, all patients received MTX and 40\% JIA-U were treated with biologics. The average number of TGC doses decreased from 3.74 at baseline to 0.72 in the 48 th month. After Friedman and the post hoc test a significant difference in the daily doses of TGC could be seen from the 12th month after application of systemic IMT. The number of daily doses of TGC as well as the degree of inflammation in AC per eye decreased over time. Using generalized linear mixed models it was shown that the treatment with biologics, but not with MTX and systemic glucocorticoids, was associated with lower 